\title{
Network Analysis Indicating the Pharmacological Mechanism of Yunpi-Qufeng- Chushi-Prescription in Prophylactic Treatment of Rheumatoid Arthritis
}

Lin Li

Zhejiang Chinese Medical University

Donghai Zhou

The Second Affiliated Hospital of Zhejiang Chinese Medical University

Qiuping Liu

Zhejiang Chinese Medical University

Dianming Li

Zhejiang Chinese Medical University

Qiao Wang

Zhejiang Chinese Medical University

Xiaowei Shi

Zhejiang Chinese Medical University

Chengping Wen

Zhejiang Chinese Medical University

Lin Huang ( $\nabla$ huanglin@zcmu.edu.cn )

Zhejiang Chinese Medical University

\section{Research Article}

Keywords: Rheumatoid arthritis (RA), inflammatory disease, Yunpi-Qufeng-Chushi-Prescription (YQCP), arthromyodynia management

Posted Date: January 25th, 2021

DOI: https://doi.org/10.21203/rs.3.rs-148147/v1

License: (c) (i) This work is licensed under a Creative Commons Attribution 4.0 International License.

Read Full License 


\title{
Network Analysis Indicating the Pharmacological Mechanism of Yunpi-Qufeng- Chushi-Prescription in prophylactic treatment of Rheumatoid Arthritis
}

Lin $\mathrm{Li}^{1, \#}$, Donghai Zhou ${ }^{2, \#}$, Qiuping Liu ${ }^{1}$, Dianming $\mathrm{Li}^{1}$, Qiao Wang ${ }^{1}$, Xiaowei $\mathrm{Shi}^{1}$, Chengping Wen ${ }^{1, *}$, Lin Huang ${ }^{1, *}$

${ }^{1}$ School of Basic Medical Sciences, Zhejiang Chinese Medical University, 548 Binwen Road, Hangzhou, Zhejiang, 310000, China.

2 The Second Affiliated Hospital of Zhejiang Chinese Medical University, Hangzhou, 310005, China.

*Correspondence and requests for materials should be addressed to C.W. (email:

wengcp@163.com) and L.H. (email: huanglin@zcmu.edu.cn).

\# These authors contributed equally to this work.

\begin{abstract}
Background: Rheumatoid arthritis (RA), is an autoimmune inflammatory disease with increasing global morbidity and high disability. Early treatment is an effective intervention to slow down joint deformation. However, as for early RA and pre-RA patients, it sometimes takes a long time to make a definite diagnosis and few guidelines have made suggestion for these suspected or early phrase individuals. Yunpi-Qufeng-Chushi-Prescription (YQCP) is an optimization of the traditional formula, Cangzhu Fangfeng Tang which is effective for arthromyodynia management.
\end{abstract}

Methods: In this study, LC-MS identify the main component of YQCP. Ingredients of the 11 herbs were collected from Traditional Chinese Medicine Integrated Database (TCMID). Targets of these ingredients were collected from two source, TCMID and PharmMapper. Microarray of 20 early untreated RA patients and corresponding health control were download from NCBI Gene Expression Omnibus (GEO) database to defined the differential expressed genes.Gene ontology analysis and KEGG enrichment analysis were carried out for the YQCP. Protein-protein interactions (PPIs) networks were constructed to identify the hub 
targets. At last, molecular docking (MD) were conducted to further verified the the possibility of YQCP for RA therapy.

Result:The study indicated that by acting on hub targets such as C3, EGFR, SRC and MMP9, YQCP may influence the mature of B cells and inhibit B cell-related IgG production, regulate oxidative stress and modulate activity of several enzymes including peroxidase and metallopeptidase to delay the occurrence and progress of RA and benefit the pre-RA or early RA patients.

Conclusion: YQCP is a potential effective therapy for prophylactic treatment of RA.

\section{Introduction}

Rheumatoid arthritis (RA), is an autoimmune inflammatory disease characterized by chronic, symmetrical arthritis and extraarticular lesions. The global incidence of RA is approximately $0.24 \%$ and RA ranks within the top 50 diseases that contribute to global disability[1]. Inflammation often occurs in facet joints of the hands, wrists and feet which would have an impact on normal life. RA is a multifactorial chronic disease and the precise aetiology is remain elusive. It is reported that heritability of RA was ranging from $15 \%$ to $60 \%$, indicating genetic factor as one of the pathogenic factors[2]. In addition, a variety of environmental factors, immune cells and cytokines, such as smoking, T cells, B cells, TLRs (Toll-like receptor) and virus are involved in the pathological process $[3 ; 4 ; 5]$.

There is a prolonged phrase in onset latency of RA when serum antibody was identified in the absence of arthralgia or synovitis. It is generally considered that early identification and treatment is of great importance in RA disease management. However, as for early RA patients, it sometimes takes months or years to make a definite diagnosis and few guidelines have made suggestion for these suspected individuals. Non-steroidal anti-inflammatory drugs help alleviate the pain but cannot delay the progression of joint destruction while disease-modifying anti-rheumatic drugs slow down joint deformity but only suggested to be applied after clinical diagnosis. Therefore, it is an urgent target to put forward medical advice for early RA patients.

Traditional Chinese Medicine (TCM), especially herbal medicine has long been used in inflammation disease including arthralgia. One of the prescriptions is Cangzhu Fangfeng Prescription which was recorded in "Su Wen Bing Ji Qi Yi Bao Ming Ji". The TCM 


\section{$3 / 13$}

rheumatologist Professor Chengping Wen inherited the academic thoughts of the formula and continuous optimized it in clinic, finally coming up wtih Yunpi-Qufeng-Chushi prescription (YQCP). YQCP is consist of 9 basal herbs (Table 1), i.e. cang zhu (Atractylodes lancea), fang feng (Saposhnikovia divaricata), qing feng teng (Sinomenium acutum), jin yin hua (Lonicera japonica), hu zhang (Polygonum cuspidatum), yi yi ren (Coix lacryma- jobi var. ma - yuen), zhi gan cao (Radix Glycyrrhizae Preparata), tu fu ling (Smilax glabra), xu chang qing (Cynanchum paniculatum) and two alternative herbs designed for relieving severe joint pain, i.e. kun ming shan hai tang(Tripterygium hypoglaucum) and tu si zi(Semen Cuscutae). Some of these herbs have been demonstrated to prevent the aggravation of RA. For instance, triptolide, an extract of kun ming shan hai tang, suppresses human synoviocyte cells mobility and promote osteoclast apoptosis[6; 7]. Our research team have demonstrated that preventive treatment of YQCP can effectively delay the occurrence RA[8]. YQCP is an effective complementary alternative therapy for suspected RA patients who is lack of treatment guideline.

Because of the multi-ingredients and multi-targets hallmark, it is complex to figure out the underlying mechanism of TCM prescriptions. However, network pharmacology exhibits its superiority in addressing this issue and the result always offers effective advice for further experimental verification[9]. In this study, network pharmacology method was applied to unveil the potential molecular mechanism of YQCP and offers effective advice for conducting further research.

\section{Materials and Methods}

\section{Liquid chromatography and mass spectrometry}

YQCP granules were produced by China Resources Sanjiu Pharmaceutical Factory. YQCP granules were ground into powder. $0.5 \mathrm{~g}$ powder were weighed for further testing. The powder was dissolved with $5 \mathrm{~mL} 80 \%$ methanol and sonicated for $90 \mathrm{~min}$ at $35 \mathrm{kHz}$ and $25^{\circ} \mathrm{C}$. After centrifugation at $3500 \mathrm{rpm}$ for $10 \mathrm{~min}, 1 \mathrm{~mL}$ supernatant was taken which was then filtered with $0.22 \mu \mathrm{m}$ filter and transferred to a $1.5 \mathrm{~mL}$ sample vial. The data of UPLC was acquired on Waters UPLC system (Waters e2695 and a 2998 PDA detector) equipped with a C18 column (Inertsil ODS-2.1x100mm, 1.6 $\mu \mathrm{m}$ ). Solvent A was acetonitrile, and solvent B comprised 0.1\% formic acid in $99.9 \%$ water. $5 \mu \mathrm{L}$ injection were eluted at $30^{\circ} \mathrm{C}$ and a flow rate of $0.3 \mathrm{~mL} / \mathrm{min}$. using the following gradient program: 0-5\% (0-2 min) solvent A, 5-100\% solvent A (2-32min). 
The mass spectrometer was operated in positive ion scanning modes with a capillary voltage of $2.5-3.0 \mathrm{kV}$.

\section{Dataset}

Ingredients of the 11 herbs were collected from Traditional Chinese Medicine Integrated Database (TCMID)[10]. Targets of these ingredients were collected from two source, TCMID and PharmMapper[11]. TCMID and PharmMapper predicted the targets from two different approach, literature mining and molecular structure. Overlapped targets from these two databases were chosen as candidate targets. RA-related genes were get from human gene database GeneCards with the filter criteria of score > 5[12].

\section{Microarray data processing of RA sample}

Microarray of 20 early untreated RA patients and corresponding health control were download from NCBI Gene Expression Omnibus database (GSE45867). Expression values were normalized by MAS 5.0 function in R program. Differentially expressed genes (DEGs) were defined as genes whose fold change values were larger than 4 and adjust $P$-value less than 0.01. Annotations of microarray platforms (GPL570-55999) was used transformed the probe into gene official name, excluding probe with missing values for further analyses. 1579 genes were finally defined as DEGs. Hclust in R were utilized to compute the clustering distance of DEGs. The heatmap for the DEGs set was drawn using pheamap package in $\mathrm{R}$.

\section{Network construction}

Protein-protien interactions (PPIs) data was download from Human Protein Reference Database and Biogrid[13]. Herbal-ingredient-targets and PPI networks were visualized by cytoscape 3.6. MCODE plugin were used to filter out sub-cluster for the whole PPIs network.

\section{GO enrichment analysis}

Gene Ontology (GO) analysis was carried out using Cluego plugin in cytoscape on immune process, molecular function and KEGG pathway for YQCP. GO Terms with p-value less than 0.01 were picked out.

\section{Molecular docking analysis}

To analyze the feasibility of the main ingredients of YQCP in interaction with hub targets, we applied molecular docking analysis. The 2D structure of the 5 main ingredients were download from Pubchem database. The crystal structure of the 9 hub targets were got from PDB database. $\mathrm{H}_{2} \mathrm{O}$ of the proteins were removed, and hydrogen atom were added in Pymol software. The docking were carried out using Autodocktools -1.5.6. Binding energy of docking 
result was compared with the original ligand and binding energy less than $-5.0 \mathrm{kcal} \cdot \mathrm{mol}^{-1}$ were defined as dependable binding.

\section{Results}

\section{LC-MS identify the main component}

5 main components were identified by LC-MS, i.e. chlorogenic acid in Lonicera japonica, polydatin in Rhizoma Polygoni Cuspidati, prim-O-glucosylcimifugin in Saposhnidoviae Radix, sinomenine in Caulis sinomenii and liquiritin in prepared Liquorice root (Figure 1, Figure S1). Batch information of each herbs were supplied in Supplementary Table 1.

\section{Ingredients and targets of YQCP}

699 ingredients from 11 herbs were collected from TCMID (Supplementary Table 2). 23 of them were shared between different herbs and the rest 676 were unique. As shown in Figure 2, Caulis sinomenii and Lonicera japonica are the nearest ingredients. In the theory of TCM, these two herbs could clear away heat and toxic which is closed to anti-inflammatory painkillers in modern medicine. The overlapped ingredients include (+)-catechin, tryptophan and $\beta$-sitosterol. Therein, (+)-catechin could inhibit inflammatory milieu through IL-1 $\beta$ signaling[14] while tryptophan metabolism is involved in the initiation and propagation of synovitis [15]. $\beta$-sitosterol could influence macrophage polarization in RA mice and reduce inflammatory response [16]. The unique ingredients represent individuality of each herbs and are often the most effective ingredients. For instance, sinomenine, the typical component of Caulis sinomenii reduces cartilage destruction through inhibiting inflammatory signaling

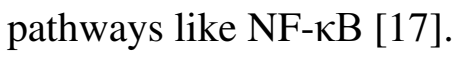

Among the 699 ingredients, 46 of them has recorded targets in TCMID, providing 1822 predicted targets. 326 of the ingredients were successfully matched to PharmMapper model, providing 3524 predicted targets. 413 targets are in common within the two database and defined as predicted formula targets, including C2, MMP2, MMP9, VEGFA. 269 ingredients associated with these targets are defined as effective compound, including triptolide, sinomenine, atractylon, coixan A and so on.

\section{Functional analysis of YQCP predicted targets}

GO analysis was carried for formula targets to predict the potential function of YQCP. On the aspect of immune system process, YQCP is mainly take part in the mature B cell 


\section{$6 / 13$}

apoptotic process and complement activation, alternative pathway on the GO level of 2 to 6 (p-value < 0.01) (Figure 3a, Table S3). Higher percentage of peripheral B cells were found in RA patients as compared to healthy control[18]. Defects in the regulation of B cell apoptosis are required for the production of CCP[19]. Complement activation might be generated in atrisk individuals in local mucosal during preclinical[20]. In molecular function, enriched GO terms include metallopeptidase activity, transmembrane receptor protein kinase activity and peroxidase activity (Figure 3b, Table S4). Excessive secretion of matrix metalloproteinases (MMPs) by fibroblast-like synoviocytes and peroxidase activity by neutrophils would lead to cartilage destruction[21;22].

Moreover, formula targets enriched in 28 signaling pathways including arachidonic acid metabolism, adherens junction and complement and coagulation cascades (Figure 4, Table S5). Cytokines released by B cells such as TNF- $\alpha$ and IL-1 $\beta$ stimulate the production of inflammatory factors of synovium and promote metallopeptidase activity[23]. Arachidonic acid metabolism pathway was demonstrated to be relate with mechanism of tocilizumab in dealing with early RA patients [24]. Adherens junction is indispensable components of the vessel wall that affect vascular permeability [25] while activation of complement and coagulation cascades modulates synovium and systemic inflammation[26]. The enrichment results suggested that function of YQCP is closely associated with RA therapy.

\section{PPI networks of finding the hub targets}

Microarray of paired synovial biopsy samples obtained before and after treatment of 20 early RA patients were analyzed to define the DEGs relevant to treatment. Firstly, quality control by qc in $\mathrm{R}$ program confirm the quality of these chips. The raw data (CEL files) was then normalized by MAS 5.0. Then the contrast was made between RA samples before and after treatment using contrasts.fit and eBays. 1579 DEGs were filtered out with fold change $>4$ and adjust $\mathrm{p}$-value $<0.01$.

Analysis result from microarray obtained 1579 DEGs. 591 RA-related genes were embodied in Genecards with score $>5.198$ proteins translated from overlapped genes in these two data were finally chosen as therapy-related target. Association between therapy-related proteins and formula targets were analyzed using PPIs network (Figure 5a) and MCODE plugin was applied to figure out the stable sub-clusters in the whole PPIs and 10 sub-clusters were meet with the criteria that cluster score $>3$ (Figure $5 \mathrm{~b}$ ). Formula targets either overlapped with disease genes or have the high degree were chosen as the candidate hub targets, such as SRC in cluster 1 and EGFR in cluster 2. Literature mining then verified the hub targets based on 
whether they contribute to the therapy or pathogenesis of RA. For instance, several recent studies demonstrated that EGFR concentrations are markedly elevated both in serum and synovial fluid in RA patients as contrasted to health controls[22]. Besides, the tissue mRNA expressions of Src kinase were increased, and it's signaling pathway is active in RA[23]. Targets like abnormal IGF-I production take effect in aberrant osteoclastic activation and angiogenesis, and IGF inhibition is beneficial for the treatment of RA[24]. As the importance of complement and MMPs is disscussed in the function analysis, we finally defined C2, C3, C5, MMP2, MMP9, SRC, KIT, IGF1R and EGFR as hub targets.

\section{Molecular docking analysis}

To ascertain the feasibility of YQCP in targeting the hub proteins, we carried out molecular docking analysis on the five identified ingredients and 9 hub targets. 2D structure of the ingredients and crystal structure of the 9 targets were downloaded. Crystal structure for C2, C3, C5, MMP2, MMP9, SRC, KIT, IGF1R and EGFR are respectively 3ERB, 3D5R, 3CU7, 1KS0, 5TH6, 3GEQ, 3G0E, 2DSP and 1JL9. Except C5 and MMP2, the rest 7 targets were all showed good affinity with the main ingredients (Table 2). Therein, combination success of sinomenine is highest among the 5 ingredients while combination success of C3 and KIT is highest among the 7 hub targets. Molecular docking diagrams of C2 - liquiritin, C3 - chlorogenic acid, IGF1R - polydatin KIT - prim-O-glucosylcimifugin and MMP9 sinomenine were showed in Figure 6.

\section{Discussion}

RA is an autoimmune disorder characterized by lasting articular inflammation and high risk for disability. DMARDS, NSAIDs are still the mainstays of therapy. Biologicals are not widespread because of the expensive medical fee. Rheumatologist have reached an agreement that early identification and treatment is of great importance in RA disease management. In China, TCM prescription has long been an alternative medicine for arthritis including RA. The superiority of TCM is prevention before disease onset.

Cangzhu Fangfeng Tang is an effective traditional formula which was recorded in "Su Wen Bing Ji Qi Yi Bao Ming Ji”. With the combination of traditiaonl medicine, modern pharmacology and clinical practice, Prof. Wen come up with a modified Cangzhu Fangfeng Tang, namely YQCP. Previously, we have demonstrated that reventive treatment of YQCP can effectively delay the occurrence RA and relieve the inflammatory response [8]. Because of the 


\section{$8 / 13$}

hallmarks of multi-ingredients and multi-targets, it is difficult to uncover the specific mechanism of TCM formula. Recently, network pharmacology and bioinformatics were widely used in predicting the molecular function, providing guidance on experiment design.

In this research, two databases, i.e. TCMID and Pharmmapper, which used different ways to predict chemical targets was applied to gather the formula targets. 413 overlapped targets in those two databases was adopted as targets of YQCP. GO analysis indicated that YQCP may take effect through three aspects: mature B cell apoptotic process, complement alternative pathway activation and enzymes activity. Overproduction of mature B cells and B cellmaturation antigen favor towards the production of ACPA contributes to the aggravation of RA[27; 28; 29]. Moreover, B cell helping capacity are elevated in seropositive of patients in early phrase of RA and B cell is an independent factor impacting curative effect[30; 31]. Complement alternative pathway activation is involved in decay-accelerating activity of B cells[32]. As shown in Figure 4, cluster 6 contains three targets, C3, C5 and C2. Therein, both C5 and C3 take part in complement alternative pathway and influence aberrant activation of B cell[33; 34]. The GO result shows that YQCP could modulate the activity of some enzymes such as endopeptidase, peroxidase and metallopeptidase. Enzyme inhibitor has been used as therapeutic agent for a long time. Different enzyme plays different roles in the pathogenesis. Among them, endopeptidase is a kind of fibroblast activation protein that takes part in remodeling of tissues at sites of inflammation. The GO item endopeptidase activity involves hub targets of YQCP MMP2, MMP9 and C3. MMP2 and MMP9 is a matrix metallopeptidase. Overexpression of MMP9 facilitate bone erosion[35]. Peroxidase concerns ROS removal. Overproduction of ROS represent the exacerbation of oxidative stress, indicating an on-going inflammation reaction[36].

In addition to GO analysis, PPIs also screen out some hub targets. SRC is a high degree formula target in cluster $1 . \mathrm{SRC}$ is a regulator of integrin-mediated adhesion that involved in bone resorption. It is reported that SRC is regulated by ROS in osteoclast differentiation[37]. The inhibitor of KIT, another hub targets in cluster 1, has been utilized for the treatment of RA[38]. EGFR concentrations are markedly elevated both in serum and synovial fluid in RA patients. EGFR transactivation contribute to cartilage destruction and EGFR inhibitor is also an common drug targets for immune disorders[39; 40]. Cluster 3 mainly effect the NF- $\kappa \mathrm{B}$ activation. Hence, C2, C3, C5, MMP2, MMP9, SRC, KIT, IGF1R and EGFR were confirmed as potential hub targets in YQCP for RA intervention.

At last, we carried out autodock to figure out the feasibility of YQCP taking effect on hub targets. The result indicated that the main ingredients of YQCP showed high affinity to the hub 
targets. Moreover, every ingredient can at least success combined with 4 hub targets which substantiated the multi-target effect of the herbs. Among them, sinomenine exhibited the highest affinity.

\section{Conclusions}

In conclusion, multi-targets and multi-ingredients is the hallmark of TCM. As showed in Figure 7, YQCP, through acting on hub targets such as EGFR, C2,MMP9, may influence the mature of B cells and inhibit B cell-related IgG production, regulate oxidative stress and modulate activity of several enzymes including peroxidase and metallopeptidase to delay the occurrence and progress of RA and benefit the pre-RA or early-RA patients.

\section{List of abbreviations}

\begin{tabular}{ll}
\hline Abbreviation & English full name \\
\hline YQCP & Yunpi-Qufeng-Chushi-Prescription \\
DEGs & Differentially expressed genes \\
RA & Rheumatoid arthritis \\
TLRs & Toll-like receptor \\
TCM & Traditional Chinese Medicine \\
GO & Gene Ontology \\
MMPs & matrix metalloproteinases \\
PPIs & Protein-protein interactions \\
\hline
\end{tabular}

\section{Declarations}

\section{Ethics approval and consent to participate}

Not applicable.

\section{Consent for publication}

Not applicable.

\section{Availability of data and material}


The datasets generated and/or analysed during the current study are available in the TCMID,

http://119.3.41.228:8000/tcmid/; Pharmmapper, http://lilab-

cust.cn/pharmmapper/index.html, GEO, https://www.ncbi.nlm.nih.gov/geo, PDB,

https://www.rcsb.org/ and Pubchem, https://pubchem.ncbi.nlm.nih.gov/.

\section{Competing interests}

On behalf of all authors, the corresponding author states that there are no conflicts of interest.

\section{Funding}

This research was funded by National Key R\&D Program of China, grant number 2018TFC1705500, National Natural Science Foundation of China (NO.82004501) \&

Foundation of Zhejiang Chinese Medicine University (2021ZZ01).

\section{Authors' contributions}

Lin Li and Donghai Zhou collected and analyzed the original data, Qiuping Liu and Xiaowei Shi carried out LC-MS, Dianming Li and Qiao Wang made molecular docking.Lin Huang and Chengping Wen designed the research and critically reviewed the manuscript. All authors approved the final version of the manuscript.

\section{Acknowledgments}

The authors thank all colleagues at the Zhejiang Chinese Medical University.

\section{References}

[1] E. Smith, D. Hoy, M. Cross, T.R. Merriman, T. Vos, R. Buchbinder, A. Woolf, and L. March, The global burden of gout: estimates from the Global Burden of Disease 2010 study. Ann Rheum Dis 73 (2014) 1470-1476.

[2] I.B. McInnes, and G. Schett, MECHANISMS OF DISEASE The Pathogenesis of Rheumatoid Arthritis. New Engl J Med 365 (2011) 2205-2219.

[3] S.M.J. Paulissen, J.P. van Hamburg, W. Dankers, and E. Lubberts, The role and modulation of CCR6+Th17 cell populations in rheumatoid arthritis. Cytokine 74 (2015) 43-53.

[4] B. Prodinger, M. Ndosi, U. Nordenskiold, T. Stamm, G. Persson, I. Andreasson, and A. Lundgren-Nilsson, Rehabilitation Provided to Patients with Rheumatoid Arthritis: A Comparison of Three Different Rheumatology Clinics in Austria, Sweden and the Uk from the Perspectives of Patients and Health Professionals. J Rehabil Med 47 (2015) 174-182.

[5] E. Sun, A. Negi, and R. Davies, An Audit of Current Clinical Practice in the Rheumatology Department of University Hospital Wales against the Top Ten Quality Standards for Rheumatoid Arthritis as Defined by the British Society for Rheumatology. Rheumatology 54 (2015) 103-103.

[6] C.M. Xie, J. Jiang, J.P. Liu, G.H. Yuan, and Z.Y. Zhao, Triptolide suppresses human synoviocyte MH7A cells mobility and maintains redox balance by inhibiting autophagy. Biomed Pharmacother 115 (2019).

[7] S.L. Wang, Z.G. Liu, J.C. Wang, Y.F. Wang, J.H. Liu, X.Y. Ji, and X.C. Wang, The triptolide-induced apoptosis of osteoclast precursor by degradation of cIAP2 and treatment of rheumatoid arthritis of TNF-transgenic mice. Phytother Res 33 (2019) 342-349. 


\section{$11 / 13$}

[8] H. Lin, Z. Donghai, H. Zhixing, W. Qiao, W. Chengping, and Y. Z., Effect of Yunpi Qufeng Chushi Prescription on CIA Rats. Journal of Zhejiang Chinese Medicine University 44 (2020) 929-934+948.

[9] R.M. Wu, B. Jiang, H. Li, W.Z. Dang, W.L. Bao, H.D. Li, G. Ye, and X. Shen, A network pharmacology approach to discover action mechanisms of Yangxinshi Tablet for improving energy metabolism in chronic ischemic heart failure. Journal of ethnopharmacology 246 (2019) 112227.

[10] L. Huang, D. Xie, Y. Yu, H. Liu, Y. Shi, T. Shi, and C. Wen, TCMID 2.0: a comprehensive resource for TCM. Nucleic acids research 46 (2018) D1117-D1120.

[11] X. Wang, Y. Shen, S. Wang, S. Li, W. Zhang, X. Liu, L. Lai, J. Pei, and H. Li, PharmMapper 2017 update: a web server for potential drug target identification with a comprehensive target pharmacophore database. Nucleic acids research 45 (2017) W356-W360.

[12] G. Stelzer, N. Rosen, I. Plaschkes, S. Zimmerman, M. Twik, S. Fishilevich, T.I. Stein, R. Nudel, I. Lieder, Y. Mazor, S. Kaplan, D. Dahary, D. Warshawsky, Y. Guan-Golan, A. Kohn, N. Rappaport, M. Safran, and D. Lancet, The GeneCards Suite: From Gene Data Mining to Disease Genome Sequence Analyses. Current protocols in bioinformatics 54 (2016) 130 1-1 3033.

[13] T.S.K. Prasad, R. Goel, K. Kandasamy, S. Keerthikumar, S. Kumar, S. Mathivanan, D. Telikicherla, R. Raju, B. Shafreen, A. Venugopal, L. Balakrishnan, A. Marimuthu, S. Banerjee, D.S. Somanathan, A. Sebastian, S. Rani, S. Ray, C.J.H. Kishore, S. Kanth, M. Ahmed, M.K. Kashyap, R. Mohmood, Y.L. Ramachandra, V. Krishna, B.A. Rahiman, S. Mohan, P. Ranganathan, S. Ramabadran, R. Chaerkady, and A. Pandey, Human Protein Reference Database-2009 update. Nucleic acids research 37 (2009) D767-D772.

[14] S. Fechtner, A. Singh, M. Chourasia, and S. Ahmed, Molecular insights into the differences in antiinflammatory activities of green tea catechins on IL-1 beta signaling in rheumatoid arthritis synovial fibroblasts. Toxicol Appl Pharm 329 (2017) 112-120.

[15] L.O. Tykocinski, A.M. Lauffer, A. Bohnen, N.C. Kaul, S. Krienke, T. Tretter, I. Adam, S.R. Mohapatra, P. Saikali, M. Lohning, M. Neidhart, S. Gay, I. Oezen, M. Platten, C.A. Opitz, and H.M. Lorenz, Synovial Fibroblasts Selectively Suppress Th1 Cell Responses through IDO1-Mediated Tryptophan Catabolism. J Immunol 198 (2017) 3109-3117.

[16] R. Liu, D.L. Hao, W.Y. Xu, J.J. Li, X.R. Li, D. Shen, K. Sheng, L. Zhao, W.W. Xu, Z.E. Gao, X. Zhao, Q.H. Liu, and Y.T. Zhang, beta-Sitosterol modulates macrophage polarization and attenuates rheumatoid inflammation in mice. Pharm Biol 57 (2019) 161-168.

[17] Y. Wu, Z. Lin, Z. Yan, Z. Wang, X. Fu, and K. Yu, Sinomenine contributes to the inhibition of the inflammatory response and the improvement of osteoarthritis in mouse-cartilage cells by acting on the Nrf2/HO-1 and NF-kappaB signaling pathways. Int Immunopharmacol 75 (2019) 105715.

[18] Jin Yang, Shaolin Zhao, Xinling Yang, Huanhuan Zhang, Ping Zheng, and H. Wu, Inhibition of B-cell apoptosis is mediated through increased expression of Bcl-2 in patients with rheumatoid arthritis. Int J Rheum Dis 19 (2016) 134-40.

[19] Marcos López-Hoyos , Regina Marquina, Esther Tamayo, Jovanna González-Rojas, Shozo Izui, Ramón Merino, and J. Merino, Defects in the regulation of B cell apoptosis are required for the production of citrullinated peptide autoantibodies in mice. Arthritis Rheum 48 (2003) 2353-61.

[20] Elizabeth A Bemis, Jill M Norris, Jennifer Seifert, Ashley Frazer-Abel, Yuko Okamoto, Marie L Feser, M Kristen Demoruelle, Kevin D Deane, Nirmal K Banda, and V.M. Holers, Complement and its environmental determinants in the progression of human rheumatoid arthritis. Mol Immunol (2019) 256-265.

[21] Seungjin Choi, Kijun Lee, Hyerin Jung, Narae Park, Jaewoo Kang, Ki-Hoan Nam, Eun-Kyeong Kim, Ji Hyeon Ju, and K.Y. Kang, Kruppel-Like Factor 4 Positively Regulates Autoimmune Arthritis in Mouse Models and Rheumatoid Arthritis in Patients via Modulating Cell Survival and Inflammation Factors of Fibroblast-Like Synoviocyte. Frontiers in immunology (2018) 1339.

[22] Anna Leichsenring, Ingo Bäcker, Paul G Furtmüller, Christian Obinger, Franziska Lange, and J. Flemmig, Long-Term Effects of (-)-Epigallocatechin Gallate (EGCG) on Pristane-Induced Arthritis (PIA) in Female Dark Agouti Rats. PLoS One 11 (2016) e0152518.

[23] H. Storch, B. Zimmermann, B. Resch, L.O. Tykocinski, B. Moradi, P. Horn, Z.A. Kaya, N. Blank, S. Rehart, M. Thomsen, H.M. Lorenz, E. Neumann, and T. Tretter, Activated human B cells induce inflammatory fibroblasts with cartilage-destructive properties and become functionally suppressed in return. Ann Rheum Dis 75 (2016) 924-932.

[24] X.M. Teitsma, W. Yang, J.W.G. Jacobs, A. Petho-Schramm, M.E.A. Borm, A.C. Harms, T. Hankemeier, J.M. van Laar, J.W.J. Bijlsma, and F.P.J.G. Lafeber, Baseline metabolic profiles of early rheumatoid arthritis patients achieving sustained drug-free remission after initiating treat-to-target tocilizumab, methotrexate, or the combination: insights from systems biology. Arthritis Res Ther 20 (2018). 
[25] M. Corr, I. Lerman, J.M. Keubel, L. Ronacher, R. Misra, F. Lund, I.H. Sarelius, and A.J. Glading, Decreased Krev Interaction-Trapped 1 Expression Leads to Increased Vascular Permeability and Modifies Inflammatory Responses In Vivo. Arterioscl Throm Vas 32 (2012) 2702-+.

[26] A.R. Crow, R. Kapur, S. Koernig, I.K. Campbell, C.C. Jen, P.J. Mott, D. Marjoram, R. Khan, M. Kim, J. Brasseit, Y. Cruz-Leal, A. Amash, S. Kahlon, I. Yougbare, H.Y. Ni, A.W. Zuercher, F. Kasermann, J.W. Semple, and A.H. Lazarus, Treating murine inflammatory diseases with an anti-erythrocyte antibody. Sci Transl Med 11 (2019).

[27] A.G. Shabgah, Z. Shariati-Sarabi, J. Tavakkol-Afshari, and M. Mohammadi, The role of BAFF and APRIL in rheumatoid arthritis. J Cell Physiol 234 (2019) 17050-17063.

[28] F. Humby, M. Bombardieri, A. Manzo, S. Kelly, M.C. Blades, B. Kirkham, J. Spencer, and C. Pitzalis, Ectopic Lymphoid Structures Support Ongoing Production of Class-Switched Autoantibodies in Rheumatoid Synovium. Plos Med 6 (2009) 59-75.

[29] F. Rivellese, D. Mauro, A. Nerviani, S. Pagani, L. Fossati-Jimack, T. Messemaker, F.A.S. Kurreeman, R.E.M. Toes, A. Ramming, S. Rauber, G. Schett, G.W. Jones, S.A. Jones, F.W. Rossi, A. de Paulis, G. Marone, M.E.M. El Shikh, F. Humby, and C. Pitzalis, Mast cells in early rheumatoid arthritis associate with disease severity and support B cell autoantibody production. Ann Rheum Dis 77 (2018) 1773-1781.

[30] P. Fortea-Gordo, L. Nuno, A. Villalba, D. Peiteado, I. Monjo, P. Sanchez-Mateos, A. Puig-Kroger, A. Balsa, and M.E. Miranda-Carus, Two populations of circulating PD-1hiCD4 T cells with distinct B cell helping capacity are elevated in early rheumatoid arthritis. Rheumatology (Oxford) 58 (2019) 1662-1673.

[31] C. Kaegi, B. Wuest, J. Schreiner, U.C. Steiner, A. Vultaggio, A. Matucci, C. Crowley, and O. Boyman, Systematic Review of Safety and Efficacy of Rituximab in Treating Immune-Mediated Disorders. Frontiers in immunology 10 (2019) 1990.

[32] V. Caudwell, F. Porteu, A. Calender, M.K. Pangburn, and L. Halbwachs-Mecarelli, Complement alternative pathway activation and control on membranes of human lymphoid B cell lines. Eur J Immunol 20 (1990) 2643-50.

[33] P.A. Nikitin, E.L. Rose, T.S. Byun, G.C. Parry, and S. Panicker, C1s Inhibition by BIVV009 (Sutimlimab) Prevents Complement-Enhanced Activation of Autoimmune Human B Cells In Vitro. J Immunol 202 (2019) 1200-1209.

[34] V. Buhe, J.O. Pers, R. Marianowski, C. Berthou, P. Youinou, and S. Loisel, Development of a Murine model to dissect the CpG-oligonucleotide-enhancement of the killing of human B Cells by rituximab. Journal of autoimmunity 34 (2010) 136-44.

[35] S.G. Romeo, K.M. Alawi, J. Rodrigues, A. Singh, A.P. Kusumbe, and S.K. Ramasamy, Endothelial proteolytic activity and interaction with non-resorbing osteoclasts mediate bone elongation. Nature cell biology 21 (2019) 430-441.

[36] X. Liu, Y. Zhu, W. Zheng, T. Qian, H. Wang, and X. Hou, Antagonism of NK-1R using aprepitant suppresses inflammatory response in rheumatoid arthritis fibroblast-like synoviocytes. Artificial cells, nanomedicine, and biotechnology 47 (2019) 1628-1634.

[37] J. Lee, H.S. Son, H.I. Lee, G.R. Lee, Y.J. Jo, S.E. Hong, N. Kim, M. Kwon, N.Y. Kim, H.J. Kim, Y.J. Lee, E.K. Seo, and W. Jeong, Skullcapflavone II inhibits osteoclastogenesis by regulating reactive oxygen species and attenuates the survival and resorption function of osteoclasts by modulating integrin signaling. Faseb J 33 (2019) 2026-2036.

[38] J. Tebib, X. Mariette, P. Bourgeois, R.M. Flipo, P. Gaudin, X. Le Loet, P. Gineste, L. Guy, C.D. Mansfield, A. Moussy, P. Dubreuil, O. Hermine, and J. Sibilia, Masitinib in the treatment of active rheumatoid arthritis: results of a multicentre, open-label, dose-ranging, phase 2a study. Arthritis Res Ther 11 (2009).

[39] R. Roskoski, Jr., Properties of FDA-approved small molecule protein kinase inhibitors. Pharmacological research 144 (2019) 19-50.

[40] C.Y. Huang, H.J. Lin, H.S. Chen, S.Y. Cheng, H.C. Hsu, and C.H. Tang, Thrombin promotes matrix metalloproteinase-13 expression through the PKCdelta c-Src/EGFR/PI3K/Akt/AP-1 signaling pathway in human chondrocytes. Mediators of inflammation 2013 (2013) 326041. 


\section{Figure Legends}

Figure 1. The molecular structure of main ingredients.(a) chlorogenic acid, (b) polydatin, (c) sinomenine, (e) prim-o-glucosylcimifugin, (f) liquiritin

Figure 2. The relationships between the herbs sharing the same compounds.

Figure 3. The gene ontology analysis of the YQCP predicted targets.

Figure 4. The KEGG enrichment analysis of the YQCP predicted targets.

Figure 5. PPIs network of YQCP and DEGs. The blue nodes stand for formula targets; the yellow nodes stand for the formula targets overlapped with DEGs; the purple nodes stand for down-expressed DEGs; the pink nodes stand for up-expressed DEGs. (a); The whole PPIs network. (b): sub-cluster with source $>3$.

Figure 6. Molecular docking patterns of main ingredients and key targets of YQCP. Figure 7. The overall mechanism of the YQCP on RA therapy. 
Figures

(a)<smiles>O=C(/C=C/c1ccc(O)c(O)c1)O[C@H]1C[C@@](O)(C(=O)O)C[C@H](O)[C@H]1O</smiles>

(b)

(c)

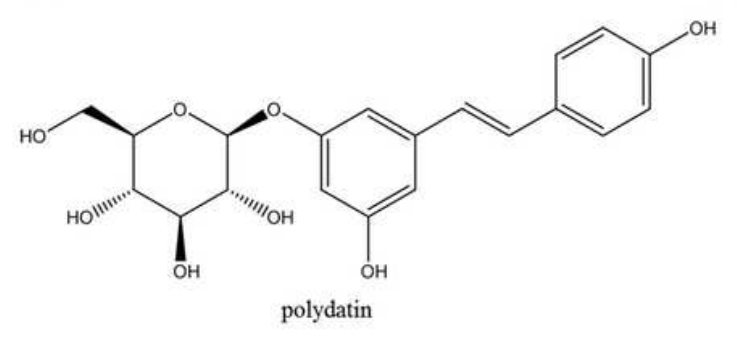<smiles>COC1=C[C@@]2(C)[C@H]3Cc4ccc(OC)c(O)c4[C@]2(C=CC3=O)CN1C</smiles>

(d)<smiles>CCCOCCCCCCCCCOc1cc(=O)c2c(OC)c3c(cc2o1)OC(C(C)(C)O)C3</smiles>

(e)<smiles>OC[C@H]1O[C@@H](Oc2cc(O)cc(/C=C/c3ccc(O)cc3)c2)[C@H](O)[C@@H](O)[C@@H]1O</smiles>

\section{Figure 1}

The molecular structure of main ingredients.(a) chlorogenic acid, (b) polydatin, (c) sinomenine, (e) prim-oglucosylcimifugin, (f) liquiritin 


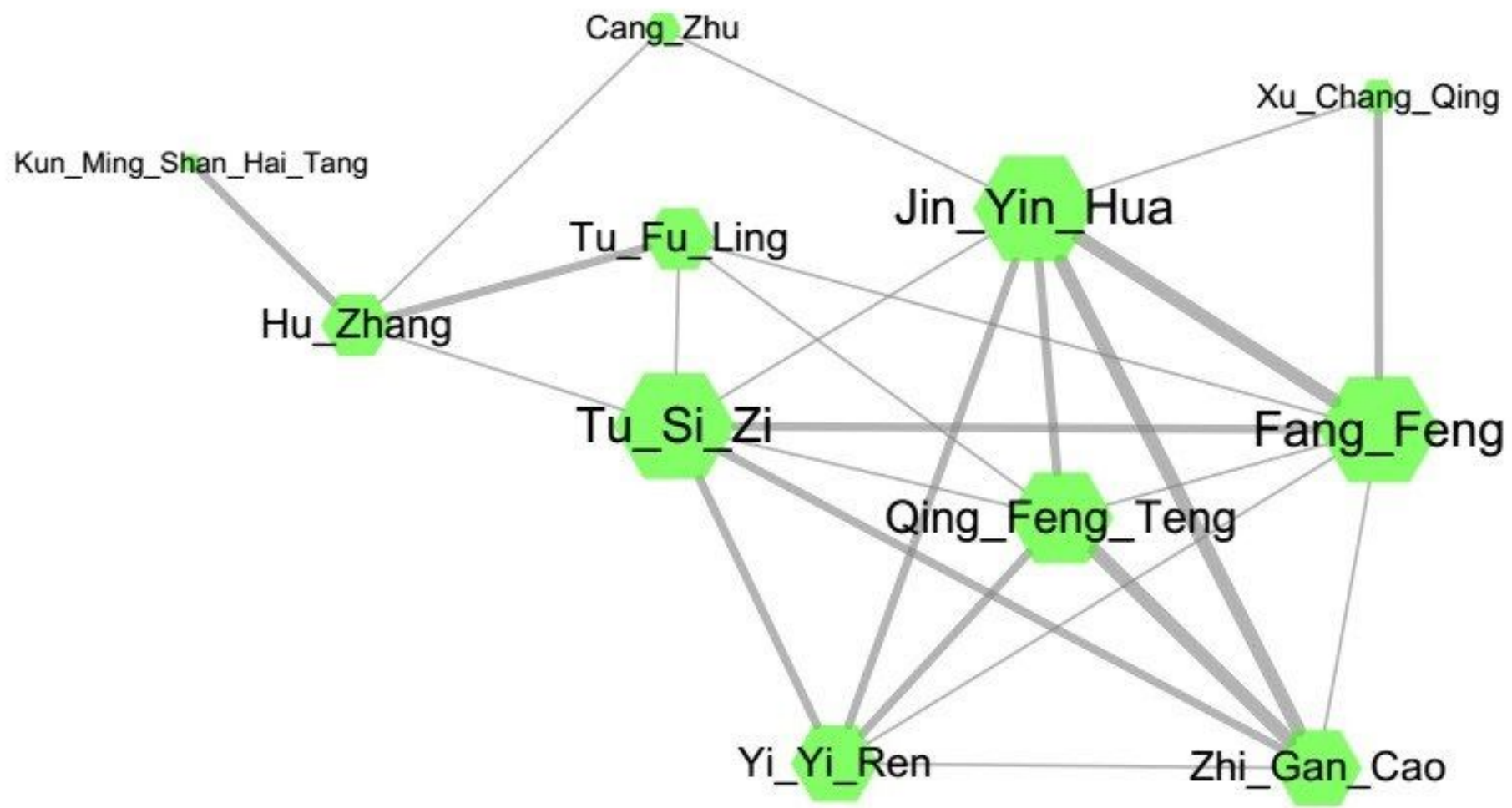

Figure 2

The relationships between the herbs sharing the same compounds.

(a)

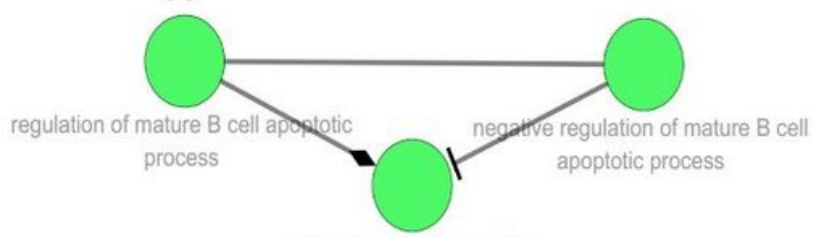

mature B cell

apoptotic process

(b)

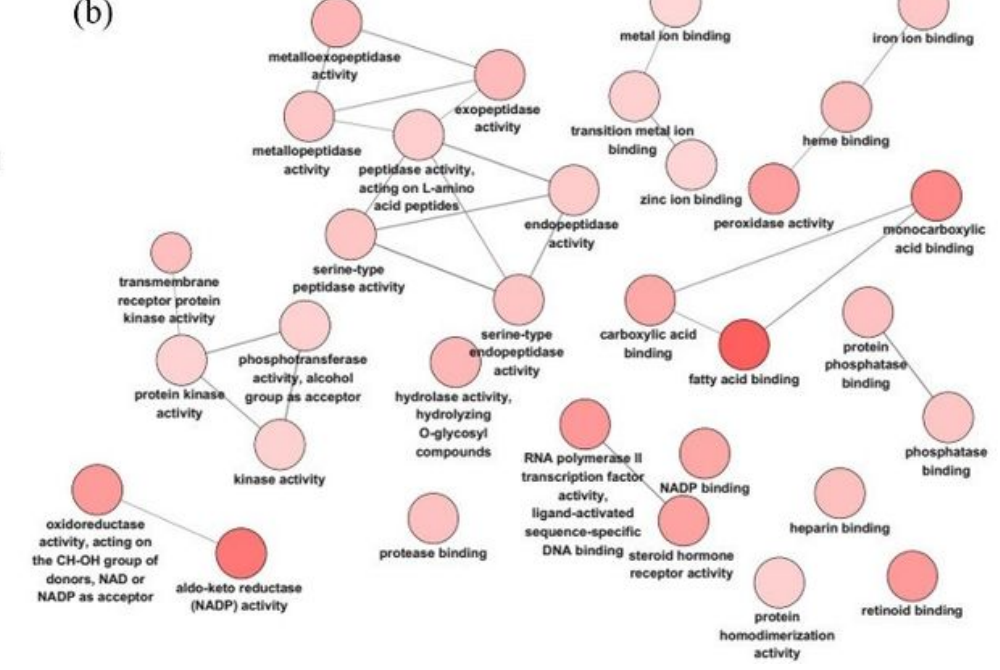

\section{Figure 3}

The gene ontology analysis of the YQCP predicted targets. 


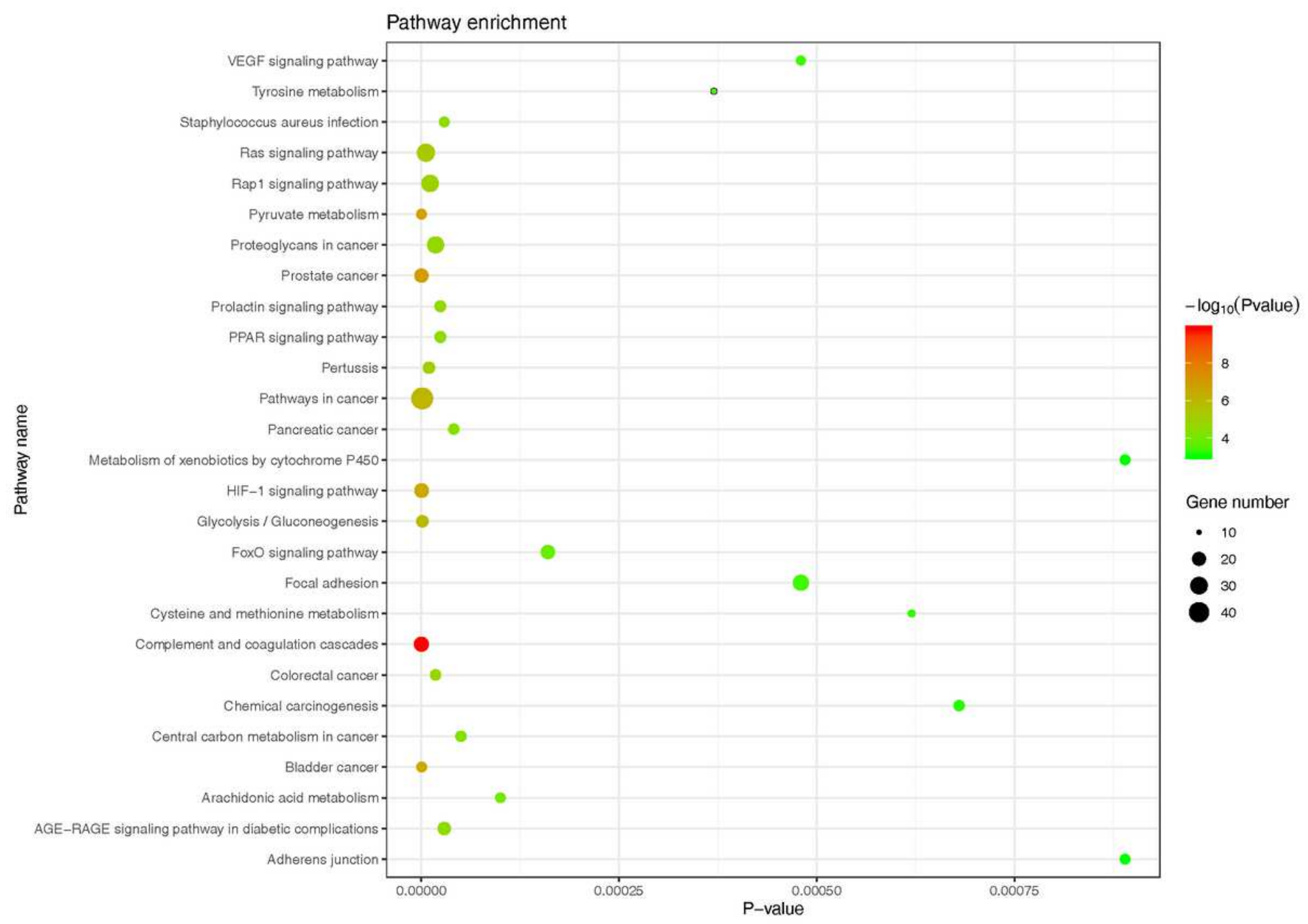

\section{Figure 4}

The KEGG enrichment analysis of the YQCP predicted targets.

(a)

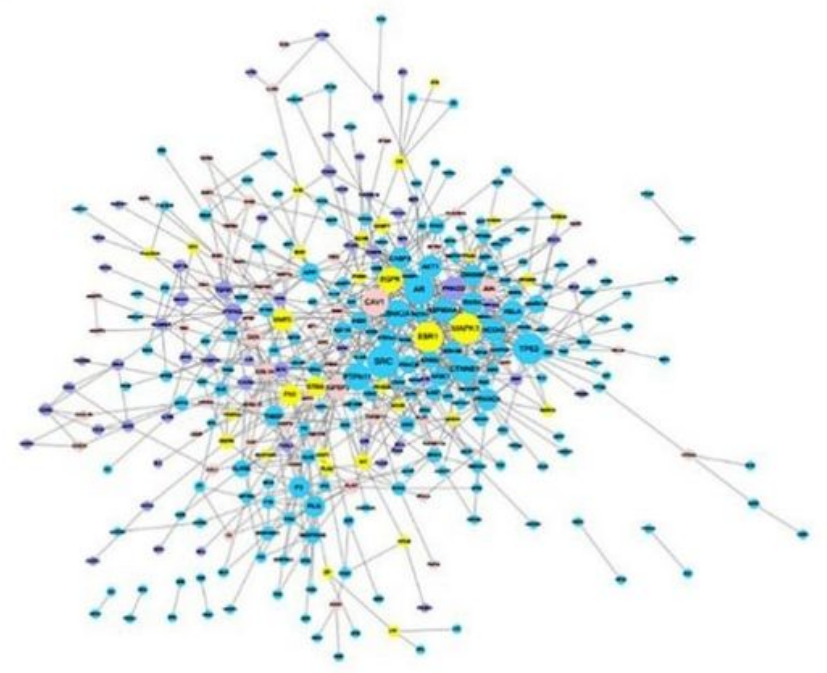

(b)

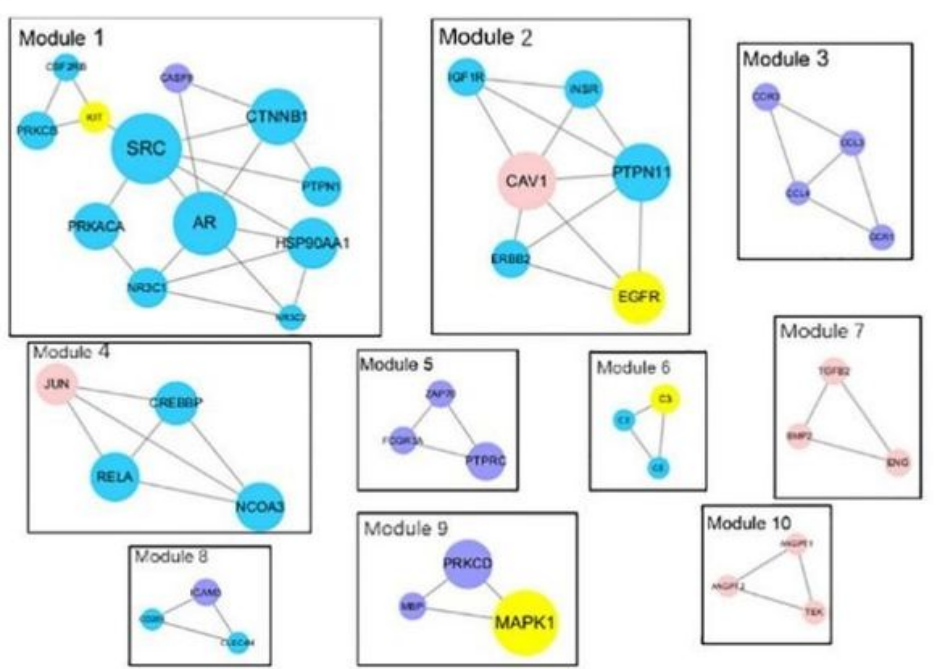


PPIs network of YQCP and DEGs. The blue nodes stand for formula targets; the yellow nodes stand for the formula targets overlapped with DEGs; the purple nodes stand for down-expressed DEGs; the pink nodes stand for up-expressed DEGs. (a); The whole PPIs network. (b): sub-cluster with source $>3$.

(a)

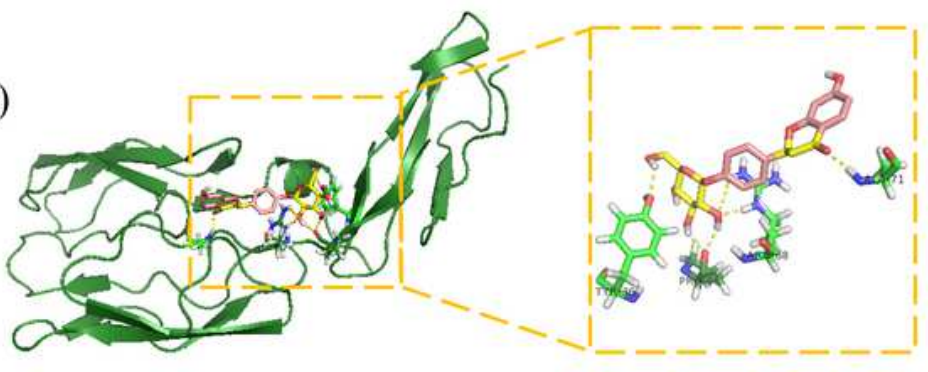

(b)

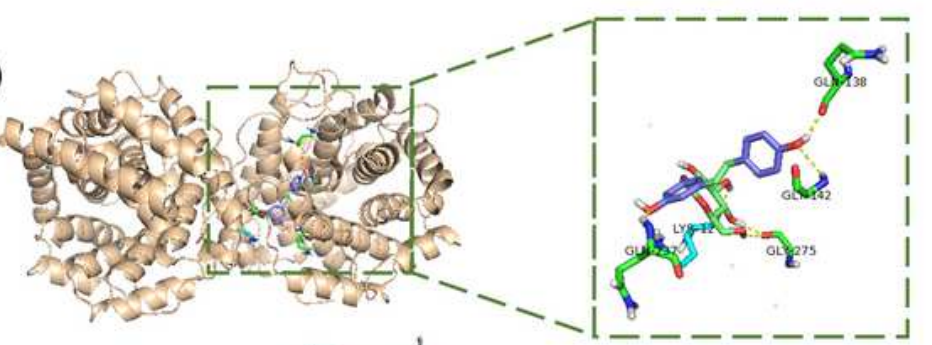

(c)

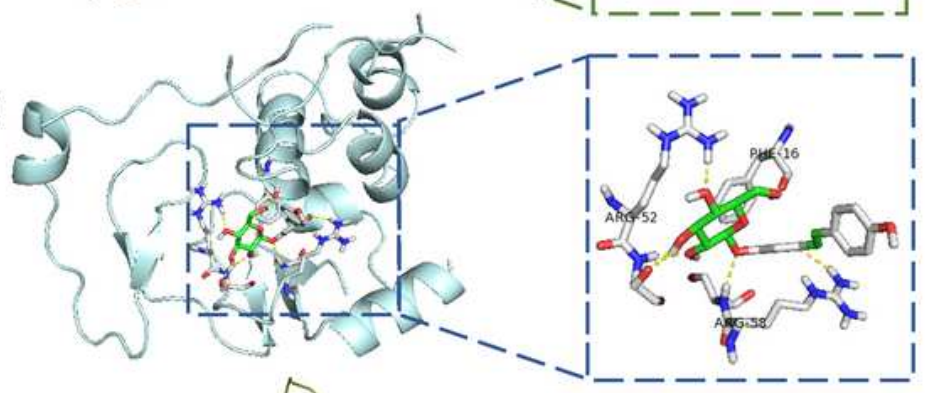

(d)

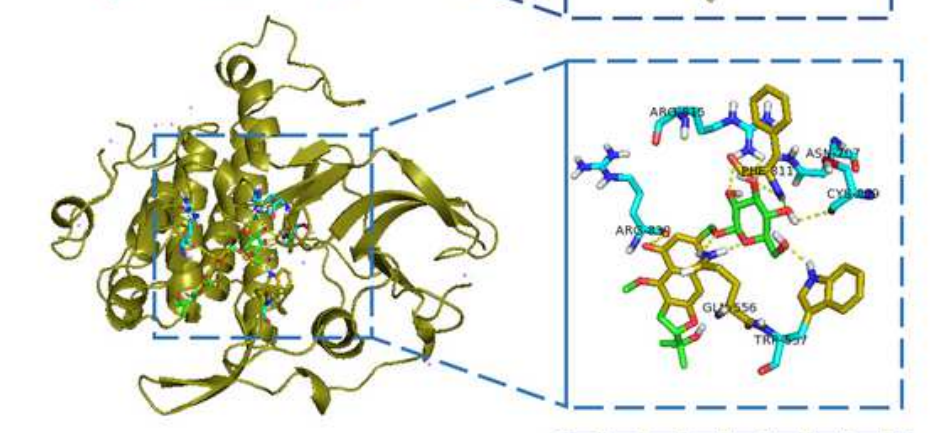

(e)

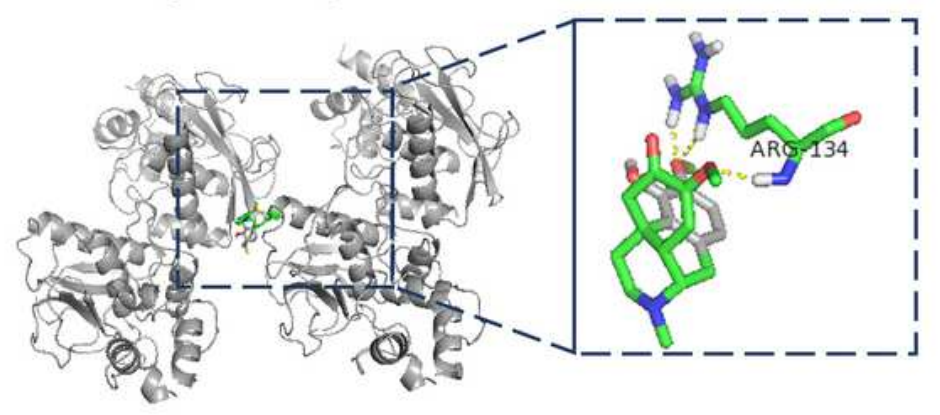

Figure 6

Molecular docking patterns of main ingredients and key targets of YQCP. 


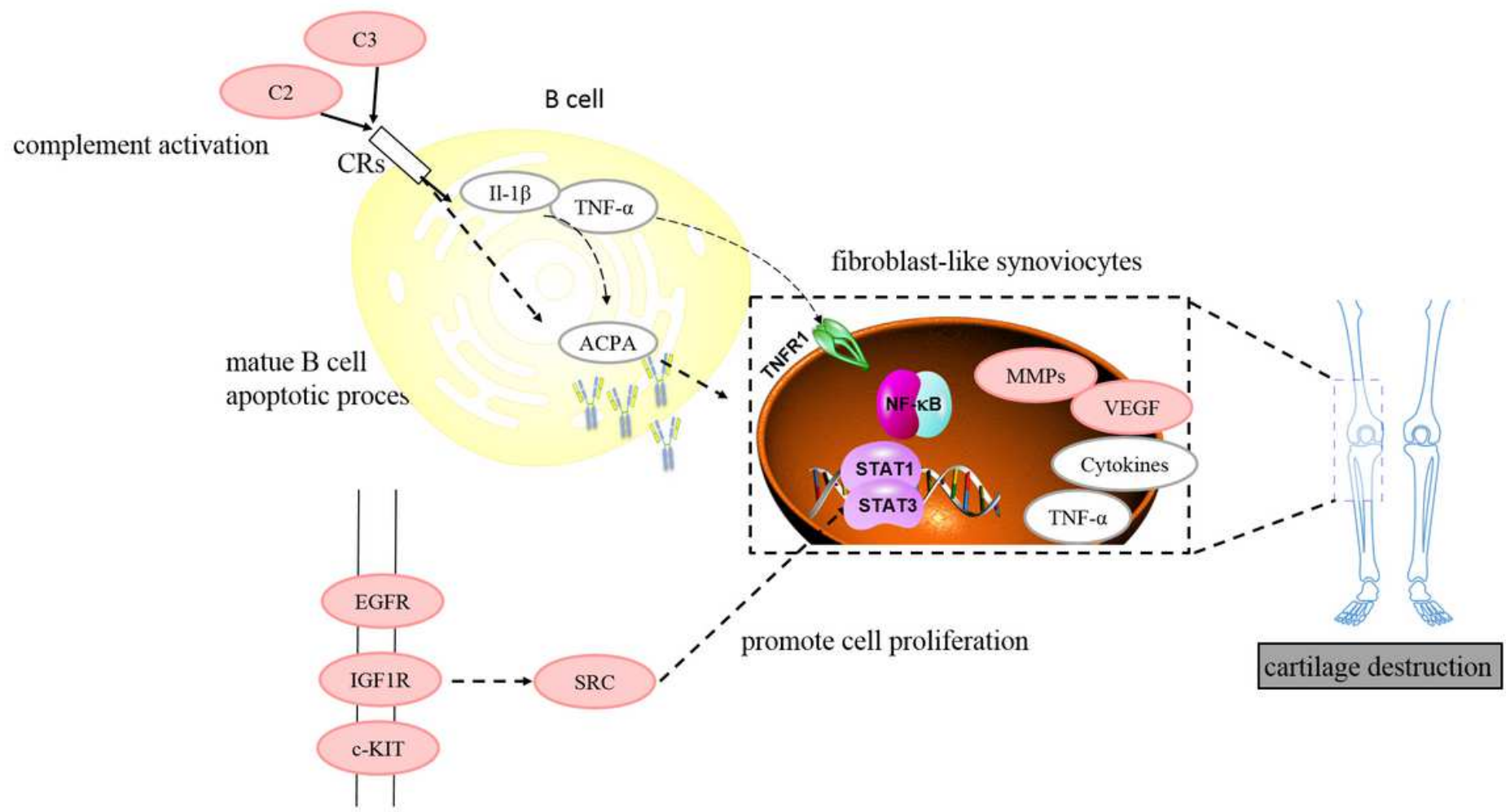

\section{Figure 7}

The overall mechanism of the YQCP on RA therapy.

\section{Supplementary Files}

This is a list of supplementary files associated with this preprint. Click to download.

- 2020s.docx

- tables12.docx 